\title{
Solid-State Recycle Processing for Magnesium Alloy Waste via Direct Hot Forging
}

\author{
Katsuyoshi Kondoh ${ }^{1}$, Tachai Luangvaranunt ${ }^{1, *}$ and Tatsuhiko Aizawa ${ }^{1}$ \\ ${ }^{1}$ Research Center for Advanced Science and Technology, The University of Tokyo, Tokyo 153-8904, Japan
}

\begin{abstract}
A solid-state recycle processing for magnesium alloy waste has been developed by combining cyclic plastic working and direct hot forging under the short thermal explosion. AZ91D machined chips, which were employed as wasted materials in this study, were consolidated to the green compact with fine microstructures via bulk mechanical alloying (BMA) process, where the compaction and forward extrusion in the closed die were repeated at room temperature. To keep fine microstructures after hot forging, that is, to prevent from the matrix softening due to the grain and/or intermetallic growth, the thermal damage on the green compact in pre-heating before forging was controlled by using the infrared gold image rapid heating furnace. The hot forged AZ91D alloy showed superior mechanical properties such as hardness and ultimate tensile strength (UTS) to the cast one used as input raw materials. The same effects were recognized in the case of wasted Al-Si alloys via this process. The developed solid-state recycle processing revealed a possibility to improve the mechanical properties of the consolidated light alloys even in employing their wasted materials.
\end{abstract}

(Received October 12, 2001; Accepted December 11, 2001)

Keywords: wasted materials, solid-state recycle, fine microstructure, hot forging, minimized thermal history

\section{Introduction}

Light alloys such as aluminum, magnesium, titanium and their alloys play important roles on the earth because of their weight reduction of the components and/or systems related to the life. For examples, they provide a possibility to reduce both the energy consumption in working and the environmental burdens (air pollutions; $\mathrm{CO}_{2}, \mathrm{NO}_{x} / \mathrm{SO}_{x}$ ). On the other hand, from a viewpoint of the environmental benign "barrier-free life system", lightweight effects on medical equipments help the aged or handicapped people who need them. Therefore, light alloys will be major materials in this century. At the same time they must be satisfied with the following items; high-performance, low cost, high productivity and recyclability. In the case of magnesium alloys, having a ultra-light weight effect, the much high energy consumption in re-melting and consolidation as a recycle process is required compared to the conventional steel or cast irons. ${ }^{1,2}$ ) In the previous study, ${ }^{3,4)}$ the solid-state recycle processing for light alloys has been established by employing bulk mechanical alloying ${ }^{5)}$ (BMA) which consists of the repeated plastic works on their wasted materials in the closed die set in the hydraulic press. The green compact after BMA process shows finer microstructures, in particular the uniform distribution of fine intermetallic compounds in the matrix. BMA process improves remarkably the mechanical properties of the green compact compared to the wasted materials. In-process measurement of the energy consumption indicates that BMA process consumes much lower energy than the re-melting and solidification, when reusing wasted magnesium and aluminum alloys. However, the green compact has not a high density and strength enough to be applied as actual components, that is, the bonding strength between primary particles is poor due to not metallurgical but mechanical joining. In this study focuses on establishing the solid-state recycle processing of wasted light alloys. The mechanical properties of the dense billet consolidated from the BMAed green compact by hot

\footnotetext{
*Graduate Student, The University of Tokyo.
}

working are examined and the effects of BMA conditions and the thermal damage in hot working on the consolidated materials are also evaluated.

\section{Experimental Procedure}

AZ91D machined chips with the size in 2-5 mm range, being same as input materials in the previous work, ${ }^{4)}$ are employed as wasted materials. The chips premixed with stearic acid lubricants are fed into the die cavity with a small extrusion ratio of $1.44(30 \mathrm{~mm} \phi / 25 \mathrm{~mm} \phi)$. The cyclic compaction and forward extrusion at room temperature cause not only the mixing of the primary chips but the fragmentation or refinement of eutectic structure and massive interdendric $\mathrm{Mg}_{17} \mathrm{Al}_{12}$ intermetallic compounds. The number of BMA cycles is $100-500$ and the other BMA conditions are same as the previous study. ${ }^{4}$ ) The grinded AZ91D alloy powder, having the mean particle size of $158 \mu \mathrm{m}$, is consolidated at room temperature to form the green compact instead of the BMAed compact in the case of hot forging not via BMA process. The BMAed green compact has superior mechanical properties due to its fine microstructures and work hardening effect of the matrix. However, the softening of the green compact easily occurs by a longer thermal explosion in hot working due to both coarsening microstructures and recovery of matrix. In other words, the minimization of thermal damage or history in pre-heating before forging on the green compact is important. In this study, the BMAed green compact is pre-heat by the infrared gold image furnace in a nitrogen gas atmosphere. After pre-heating the compact, it is immediately consolidated to full density by direct hot forging process. The heating temperature and holding time in pre-heating is $723 \mathrm{~K}$ and $0.12-1.8 \mathrm{ks}$, respectively. The heating rate is $3.6 \mathrm{~K} / \mathrm{s}$, which is much larger than conventional heating by the electric tube furnace $(\sim 0.1 \mathrm{~K} / \mathrm{s})$. The green compact is consolidated in the SKD11 steel die, which size is $35 \mathrm{~mm} \phi$ and temperature is $473 \mathrm{~K}$. The forging pressure of $784 \mathrm{MPa}$ is applied by $1000 \mathrm{kN}$ hydraulic press. Figure 1 shows an appearance of hot forged AZ91D billet. The opti- 


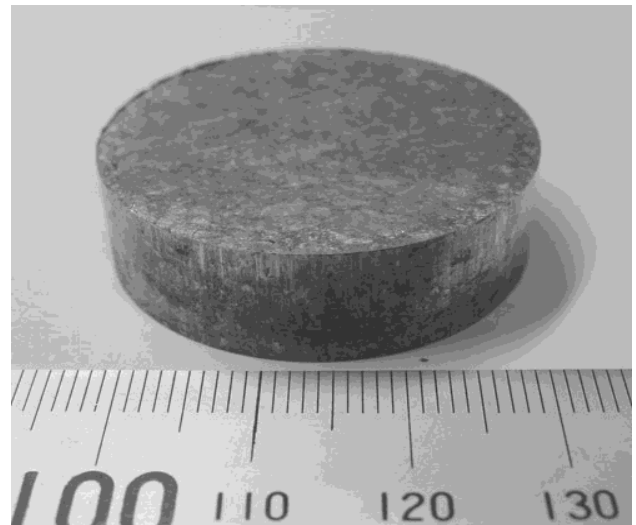

Fig. 1 Appearance of direct hot forged AZ91D alloy billet via BMA process.

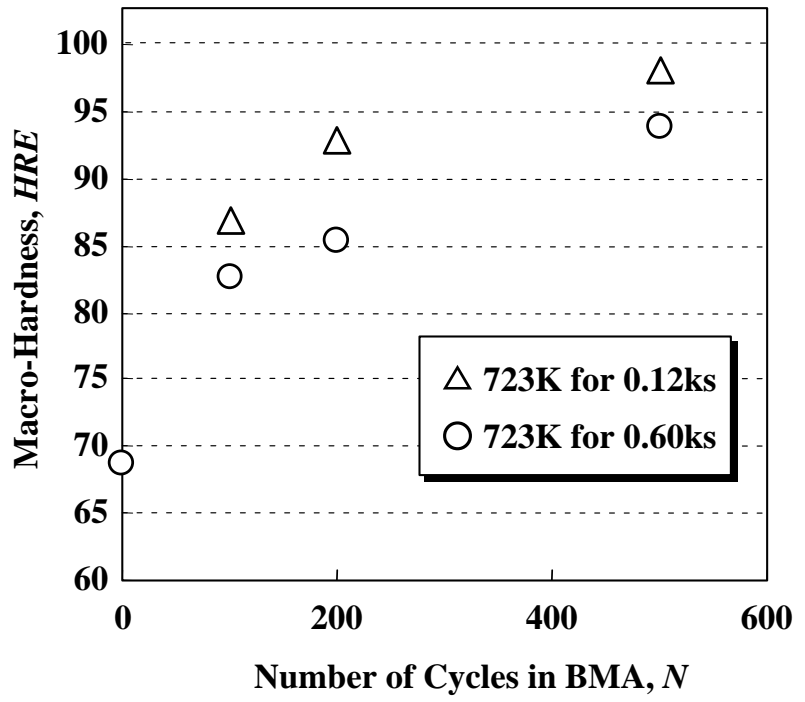

Fig. 2 Dependence of macro-hardness of direct hot forged AZ91D alloys on number of cycles in BMA process.

cal microstructures, micro/macro-hardness and ultimate tensile strength are examined. The structural evolution of the green and hot forged billets is monitored by X-ray diffraction (XRD). The fractured surface of the tensile specimen after testing is observed by scanning electron microscopy (SEM).

\section{Results and Discussion}

The previous results reveal the remarkable effect of the cycle number in BMA process on the micro-hardness $(\mathrm{Hv})$ of the green compact. This is because the repeated plastic work on the wasted light alloys causes both the refinement of microstructures and the work hardening. It can be predicted that the number of BMA cycles is also effective on the mechanical properties after hot forging. Figure 2 shows the dependence of the macro-hardness (HRE) of the direct hot forged AZ91D alloys on the number of BMA cycles. Two kind of the holding time conditions are employed; $0.12 \mathrm{ks}$ and $0.6 \mathrm{ks}$ at $723 \mathrm{~K}$, respectively. The relative density of each forged specimen is more than $99 \%$. Macro-hardness decreases gradually with increase in the number of BMA cycles. The values are much higher than that of the forged AZ91D alloy not via BMA process. The effect of refining microstructures by
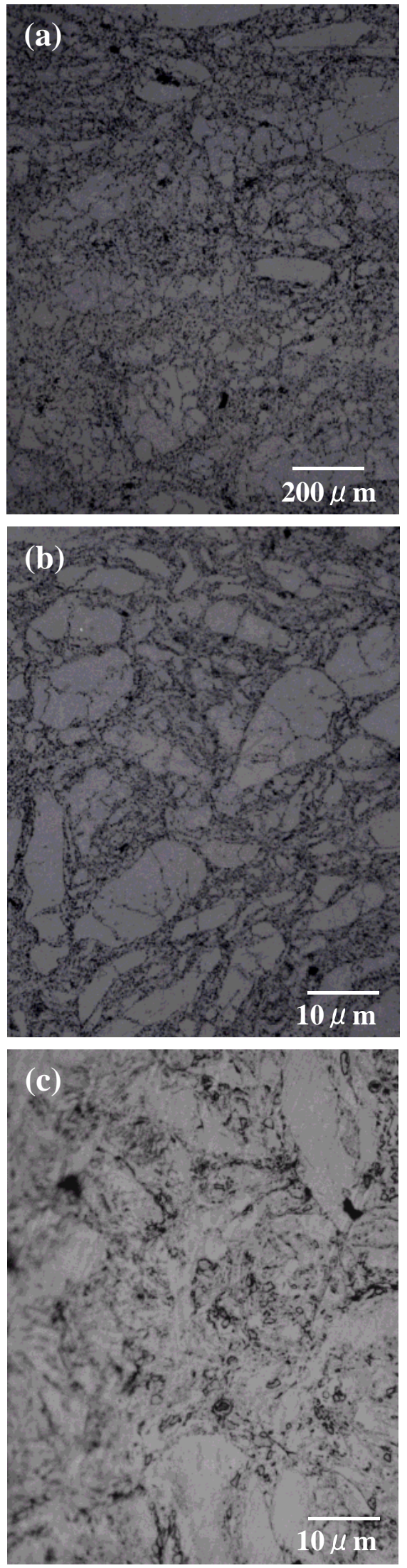

Fig. 3 Optical microstructures of AZ91D alloys, as-hot forged specimen after pre-heating at $723 \mathrm{~K}$ for $0.6 \mathrm{ks}$ (a) and (b), and as-BMAed green compact after 200 cycles (c). 


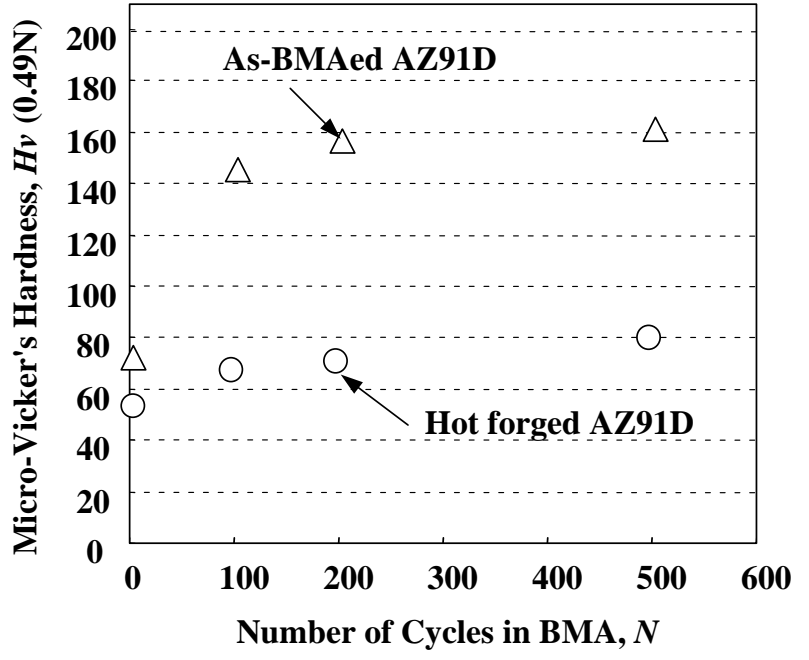

Fig. 4 Dependence of micro-hardness of as-BMAed green compact and hot forged AZ91D billet on number of BMA cycles.

cyclic plastic works on the mechanical properties of forged alloys is recognized. It also reveals that the macro-hardness decreases by a longer holding time in pre-heating. Figure 3(a) and (b) show the microstructures of hot forged AZ91D, when applying the cycle number of 200 and the pre-heating condition at $723 \mathrm{~K}$ for $0.12 \mathrm{ks}$. The primary particle boundaries, which are observed in the BMAed green compact shown in Fig. 3(c), and the gaps between particles are not clear after hot forging, that is, the hot working provides the metallurgical good bonding between the grains in $10-30 \mu \mathrm{m}$ range. Intermetallic compounds are distributed uniformly in the matrix, which correspond to "dark gray fine dots" shown in Fig. 3(b). As characterized in Fig. 4, the micro-hardness of hot forged AZ91D alloys is $68-81 \mathrm{Hv}$, and is higher than that in the case of not via BMA process. It increases gradually with increase in the cycle number. However, their values are about half of the as-BMAed materials, that is, the remarkable decrease of the micro-hardness occurs by pre-heating and hot forging. This is because of the softening of the alloys due to the recovery of the work-hardened matrix and the coarsening of $\mathrm{Mg}_{17} \mathrm{Al}_{12}$ intermetallic compounds. Figure 5 reveals changes in the XRD patterns of consolidated AZ91D alloys before (as-BMAed) and after pre-heating (as-forged). Before pre-heating shown in Fig. 5(a), the peak intensity of $\mathrm{Mg}_{17} \mathrm{Al}_{12}$ intermetallic compounds decreases with increase in the number of BMA cycles, that is, it means the fragmentation and refinement of coarse eutectic intermetallics. After pre-heating in Fig. 5(b), $\mathrm{Mg}_{17} \mathrm{Al}_{12}$ peak is detected in every material, irrespective of the cycle number. There is not significant difference of the peak intensity for each alloy. It means the precipitation or growth of the intermetallic compounds in the matrix by the thermal explosion.

The thermal history in pre-heating is certainly effective on the mechanical properties of hot forged alloys. Concerning the influence of the thermal explosion in pre-heating on them, the micro-hardness of the forged AZ91D alloys decrease during a longer explosion at $723 \mathrm{~K}$ as shown in Fig. 6. However, they are remarkably high compared to the pre-heated material by the conventional electric tube furnace, that is, the rapid pre-heating is dominates on the mechanical properties. Figure 7 shows the relationship between the ultimate tensile
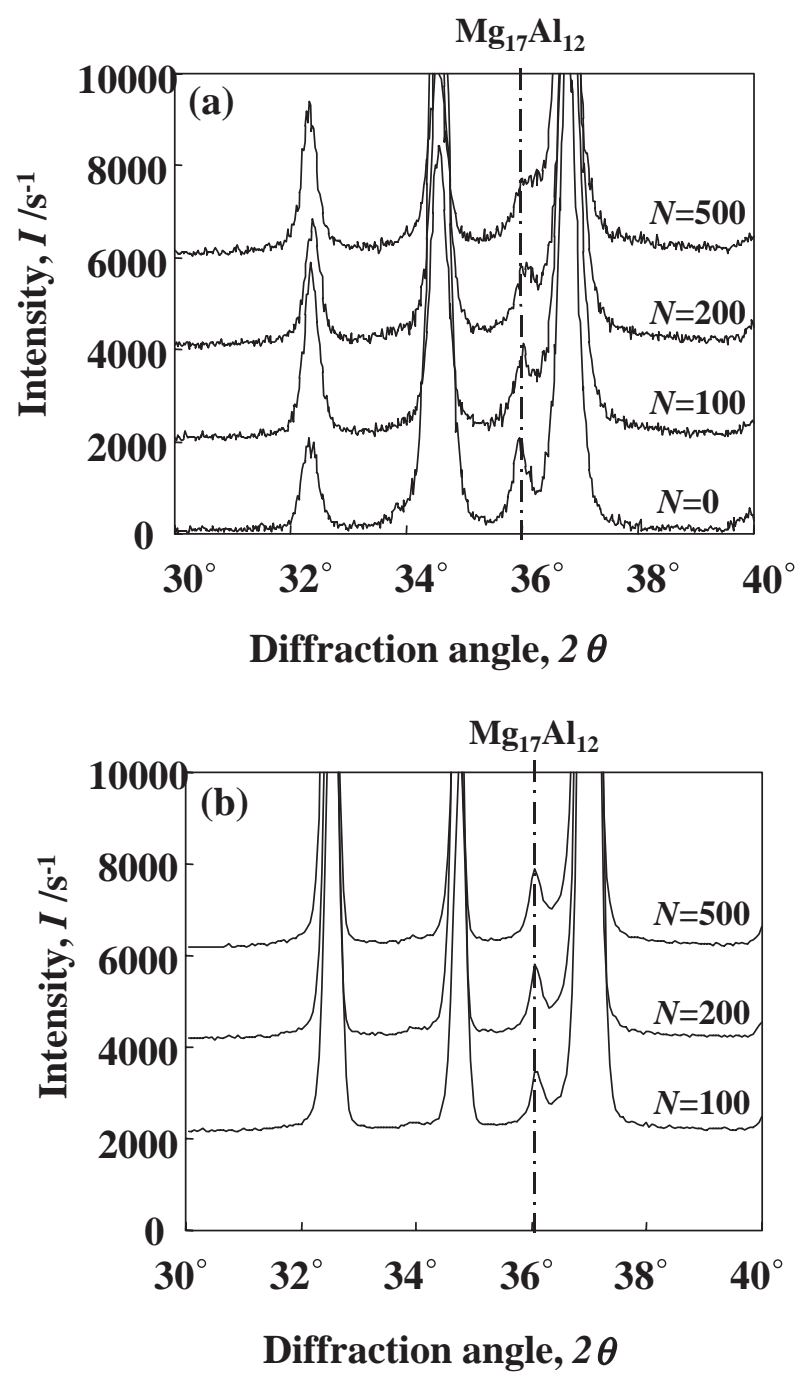

Fig. 5 Changes in XRD patterns of as-BMAed AZ91D green compacts (a) and its hot forged specimen (b).

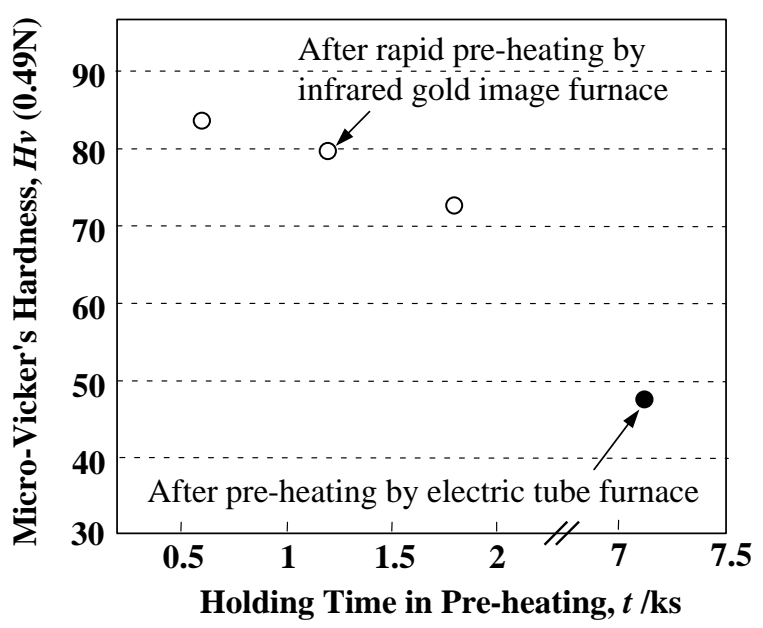

Fig. 6 Influence of holding time in pre-heating at $723 \mathrm{~K}$ on micro-hardness of hot forged AZ91D alloys via BMA process with 200 cycles.

strength and the holding time in pre-heating and SEM observation on the fractured surface of the tensile specimens. The UTS also shows the same dependence on the holding time as the micro-hardness shown in Fig. 6. The values after hot forging, however, are superior to the as-cast AZ91D alloy. In other words, this recycle process provides "rank-up service" 

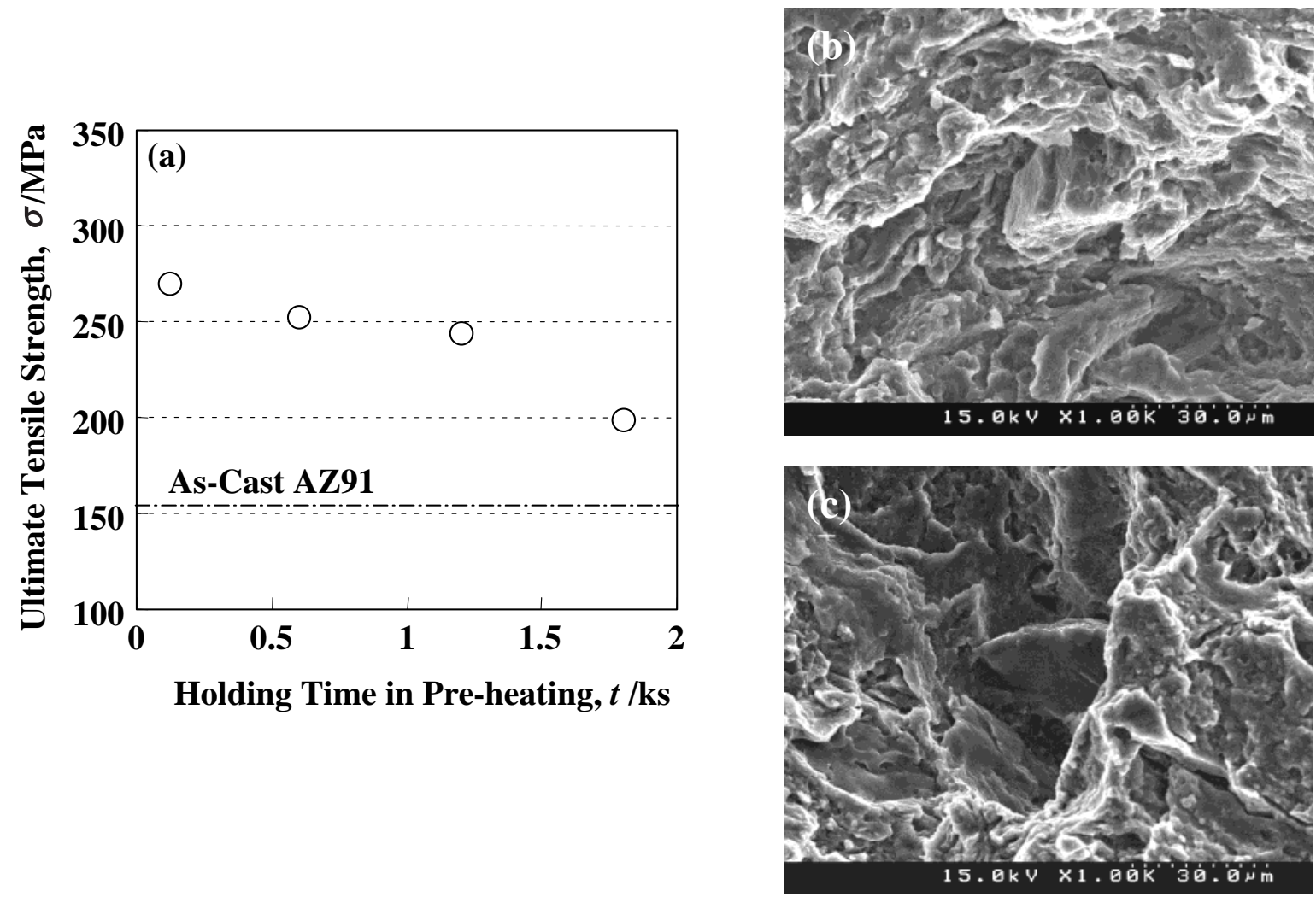

Fig. 7 Influence of holding time in pre-heating on UTS of direct forged AZ91D alloys via BMA process with 200 cycles (a), and SEM observation on fractured surface of tensile specimen pre-heat at $723 \mathrm{~K}$ for $0.12 \mathrm{ks}$ (b) and $1.8 \mathrm{ks}$ (c).

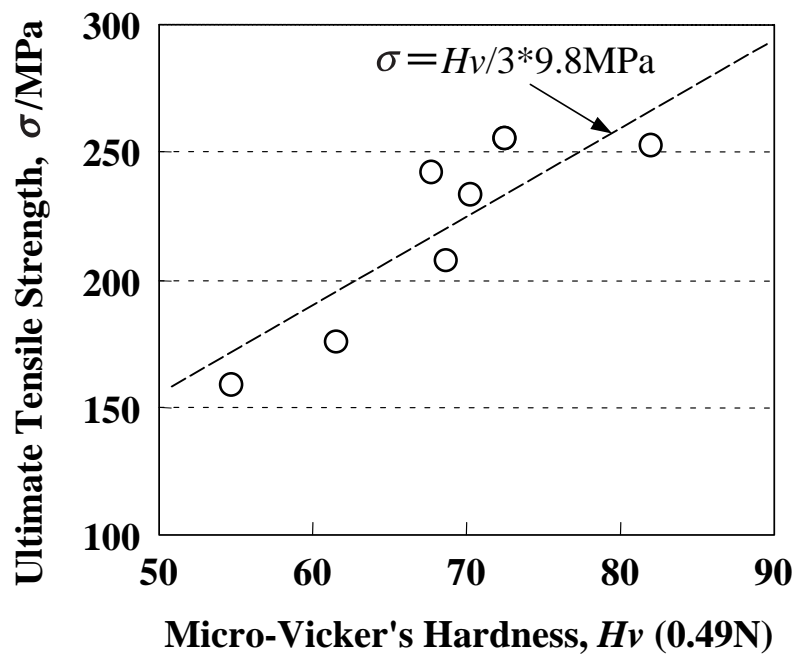

Fig. 8 Relationship between micro-hardness and UTS of direct forged AZ91D alloys via BMA process.

for high performance even in employing wasted materials. The SEM observation results also indicate that the AZ91D alloy consolidated under a shorter holding time of $0.12 \mathrm{ks}$ has small dimples and/or grains compared to the forged one with a longer thermal explosion of $1.8 \mathrm{ks}$. Figure 8 shows the relationship between the micro-hardness and UTS values of hot forged AZ91D alloys, where the empirical rule of ingot metallurgy magnesium alloys ${ }^{6)}$ is expressed as a broken line. Each data is located near the line, that is, the hot forged AZ91D alloys produced from the wasted chips has a good metallurgical bonding between primary particle boundaries.

\section{Conclusion}

To establish the solid-state recycle processing of magnesium alloys via BMA and direct hot forging processes, the influences of the BMA process conditions and the thermal damage in pre-heating on the mechanical properties were discussed. The refinement of microstructures by BMA is still effective on them even after hot forging. The minimization or optimization of the thermal history is necessary to produce the forged materials with superior mechanical properties. The wasted AZ91D chips can be reused in forming higher performance materials via this solid-state recycle processing under the small thermal explosion.

\section{Acknowledgements}

The authors gratefully acknowledge the financial support from the project "Barrier-Free Processing of Materials for Life-Cycle Design for Environment" by Science and Technology Agency.

\section{REFERENCES}

1) L. Riopelle: JOM 19 (1996) 44-46.

2) T. Aizawa, T. Luangvaranunt and K. Kondoh: Proc. Symp. Envir. Conscious Mat.-Ecomat., (CIM-2000, 2000) pp. 273-288.

3) T. Aizawa, T. Luangvaranunt and K. Kondoh: J. Japan Inst. Metals 65 (2001) 581-588.

4) K. Kondoh, T. Luangvaranunt and T. Aizawa: Mater. Trans. 42 (2001) 1254-1257.

5) O. Kobayashi, T. Aizawa and J. Kihara: Mater. Trans., JIM 37 (1996) 1497-1504.

6) J. A. Hawk, R. E. Franck and H. G. F. Wilsdorf: Metall. Trans. A 19 (1988) 2363-2366. 